\title{
A Second Cryoballoon System- new and improved?
}

\author{
Wilber $\mathrm{Su}^{1}$ \\ ${ }^{1}$ Banner - University Medical Center Phoenix
}

February 10, 2021

\begin{abstract}
Generations of cryoballoon transformed the atrial fibrillation ablation landscape. New advancements continue to make cryoballoon more successful and safer treatment. A new cryoballoon PolaRx from Boston Scientific has unique features compared to that of the Medtronic Arctic Front Advance system. Comparison of the two available cryoballoons will require ongoing larger trial and clinical experience.
\end{abstract}

\section{A Second Cryoballoon System- new and improved?}

Wilber W. Su MD $\mathrm{MD}^{1,2}$

Banner-University Medical Center, Phoenix, AZ

Stanford University Medical Center, Palo Alto, CA

Disclosures: Dr Su has received consulting fees, honoraria, and research grants from Medtronic and Boston Scientific.

Funding: None

Cryoballoon as a single-shot pulmonary vein (PV) isolation tool with the Arctic Front ${ }^{\mathrm{TM}}$ Cryoablation System has been popularized world-wide, with over 700,000 cases performed to date and vast clinical experience and expanded indications. ${ }^{1}$ Technique, dosing, and best practices to avoid complications has also improved with the Arctic Front Advance cryoballoon over the past 15 years. ${ }^{2,3}$ A second cryoballoon is now in clinical use with initial approval in EU and ongoing IDE trial in the U.S. The design similarities between the Medtronic Arctic Front Advance ${ }^{\mathrm{TM}}$ versus the Boston Scientific PolaRx ${ }^{\mathrm{TM}}$ are noticeable - similar delivery mechanism, sheath size, cryoballoon shape, size, and refrigerant. Is the new Boston Scientific system comparable or improved compared to that of the existing Medtronic system?

Crete et al reports their first experience of the two cryoballoon systems. Scope of the manuscript describes the procedural and clinical outcome of AF ablation in a small cohort of 40 patients, but it does offer the initial glimpse into the broad differences or lack thereof between the two systems. While the initial experience and reported outcome is generally good, with an acute procedural success and complication rate. Importantly, either system was able to produce a near $100 \%$ acute PV isolation with a mean procedure time of 60 minutes. Complications rate are minimal, and all are testament to the technology that has become reproducible in different operator's hands for a short, successful, PV isolation with low risk.

Importantly, significant differences in the biophysical parameters (i.e. rate of cooling, cryoballoon nadir temperatures, thaw times) during cryoablation with the two technologies were reported. Biophysical characteristics during ablation with the Arctic Front Advance cryoballoon have been carefully evaluated via pre-clinical studies, computational modeling, and randomized controlled trials over the last 15 years and have been used to define dosing algorithms and best practices. ${ }^{2-5}$ Despite the similarities between the two 
cryoballoons, results from this study suggest the PolaRx balloon has unique biophysical parameters and may need ongoing investigation to optimize dosing.

Significant differences do exist between the two system that is not discussed in detail, most noteworthy is the cryoballoon ablation pressure: the Medtronic cryoballoon inflation pressure is low(approximately 3 psi. After the initiation of the ablation, pressure increases up to six-times the inflation pressure and makes the cryoballoon more rigid/less-compliant and slightly increases the cryoballoon size. Compared to the Boston Scientific design, the inflation pressure of the cryoballoon stays fairly constant and low even during the ablation phase. Aside from the minor differences in the refrigerant flow rate at different phases of the ablation process, this pressure difference does significantly change how the end-user uses the cryoballoon to engages the pulmonary vein antrum: 1) Cryoballoon-PV antrum contact does not change; what one see during venogram is what one gets during ablation. There is no need for the "relook angiogram". This may lead to a more consistent and reproducible result of single-shot PV isolation. 2) "Proximal-seal technique" used to engage the antrum of the PV will yield a different effect. Can the more compliant system inadvertently allow the user to produce a less desirable lesion inside the PV? Could the transient phrenic injury rate be different due to the balloon compliance difference? 3) Segmental isolation needs may also be different, and the ability to produce extra-PV antral lesion for large area modification may also differ in efficacy. Larger studies will be needed to delineate the differences between the two cryoballoon ablation pressure for PV occlusion and single-shot PV isolation, while also establishing a safe procedure with a more compliant cryoballoon.

Many consider the success rate to be the most important outcome of the procedure. While the study groups are small in this study and can be difficult to delineate any significant differences. I find patients and myself to be more concentrated on the complication rate as the most important outcome of the procedure- success story of a procedure of course do focus on a great clinical outcome, but avoidance of major complications for any of our AF treatment remains paramount. Compared to studies of yesteryears, procedure time of 1-2 hours is now standard, and many centers are now aiming for the same-day discharge for straight-forward PV isolation. This speaks to the value of PV isolation as the first-line therapy for paroxysmal and persistent $\mathrm{AF}$.

New advancement such as variable cryoballoon size, compliance, and refrigerant distribution will continue to make cryoballoon a more successful, but more importantly safer, procedure for the treatment of AF. As several companies are now working on their own cryo-platform around the world, and this will continue to improve our success and complication rate. As with any new procedure, what lingers on most operator's mind is ability to minimize the complication rate establishing dosing and handling technic that may be unique to each of the cryo platform to maintain procedural safety.

\section{REFERENCES}

1. Calkins H, Hindricks G, Cappato R, et al. 2017 HRS/EHRA/ECAS/APHRS/SOLAECE expert consensus statement on catheter and surgical ablation of atrial fibrillation. Heart Rhythm. 2017; 14(10): e275-e444. doi: 10.1016/j.hrthm.2017.05.012.

2. Su W, Kowal R, Kowalski M, et al. Best practice guide for cryoballoon ablation in atrial fibrillation: The compilation experience of more than 3000 procedures. Heart Rhythm. 2015; 12(7): 1658-66.

3. Su W, Aryana A, Passman R, et al. Cryoballoon Best Practices II: Practical guide to procedural monitoring and dosing during atrial fibrillation ablation from the perspective of experienced users. Heart Rhythm. 2018; 15(9): 1348-1355.

4. Kuck K, Brugada J, Albenque J. Cryoballoon or Radiofrequency Ablation for Atrial Fibrillation. N Engl J Med. 2016 Sep 15;375(11):1100-1. doi: 10.1056/NEJMc1609160. PMID: 27626535.

5. Getman MK, Wissner E, Ranjan R, Lalonde JP. Relationship between time-to-isolation and freeze duration: Computational modeling of dosing for Arctic Front Advance and Arctic Front Advance Pro cryoballoons. J Cardiovasc Electrophysiol. 2019 Nov;30(11):2274-2282. doi: 10.1111/jce.14150. Epub 2019 Sep 17. PMID: 31502304; PMCID: PMC6899473. 